\title{
Argumentation schemes in AI: A literature review. Introduction to the special issue
}

\author{
Fabrizio Macagno \\ Faculdade de Ciências Sociais e Humanas, Universidade Nova de Lisboa, Lisbon, Portugal \\ E-mail:fabrizio.macagno@fcsh.unl.pt
}

Argumentation schemes $[35,80,91]$ are a relatively recent notion that continues an extremely ancient debate on one of the foundations of human reasoning, human comprehension, and obviously human argumentation, i.e., the topics. To understand the revolutionary nature of Walton's work on this subject matter, it is necessary to place it in the debate that it continues and contributes to, namely a view of logic that is much broader than the formalistic perspective that has been adopted from the 20th century until nowadays. With his book Argumentation schemes for presumptive reasoning, Walton attempted to start a dialogue between three different fields or views on human reasoning - one (argumentation theory) very recent, one (dialectics) very ancient and with a very long tradition, and one (formal logic) relatively recent, but dominating in philosophy. Argumentation schemes were proposed as dialectical instruments, in the sense that they represented arguments not only as formal relations, but also as pragmatic inferences, as they at the same time depend on what the interlocutors share and accept in a given dialogical circumstance, and affect their dialogical relation.

In this introduction, the notion of argumentation scheme will be analyzed in detail, showing its different dimensions and its defining features which make them an extremely useful instrument in Artificial Intelligence. This theoretical background will be followed by a literature review on the uses of the schemes in computing, aimed at identifying the most important areas and trends, the most promising proposals, and the directions of future research.

\section{Argumentation schemes and the dialectical paradigm}

Argumentation schemes for presumptive reasoning at the same time continued a very long and established philosophical tradition and challenged the 20th century logical paradigm. The analysis of the structure and the types of natural arguments and the ways to attack them was one of the crucial teachings of the Sophists [64,70], and the core of Aristotle's dialectical and rhetorical works, the Topics, the Rhetoric, and the Sophistical Refutations [2-4]. Under the label of "topic," Aristotle examined different types of inference links that guarantee the plausibility of the passage from one or more premises to a controversial claim [40]. These links were analyzed in the dialectical tradition as underlying a type of reasoning essentially distinct from formal syllogisms. While the latter are based on rules governing the 
relationship between the position of the terms or the propositions (or quantifiers), the passages from the premises to conclusions in dialectical syllogisms (or arguments, according to the original meaning of the term) are based on conditionals (generalizations, or maximae propositiones) that are generally known, shared, or accepted, namely endoxa (Abaelardus Dialectica, 262). The Aristotelian and dialectical perspective thus related the evaluation of arguments to the contexts in which they are used: the acceptability of arguments was regarded as depending on the type of dialogue, the contextual conventions conventions, and the audience [80]. This pragmatic perspective was neglected, even though never ignored, after Port Royal Logic. Topics were regarded as merely instruments for finding arguments (thus forgetting their essential and primary guarantee function, see [22]), and then claimed to be useless, and even "damaging to our judgment" (Arnauld \& Nicole Logic or the Art of Thinking: chap. 17 [5]). The study of the topics, once the backbone of the "logic" (dialectics), was replaced by a purely semantic and formalistic analysis of reasoning, abstracting arguments from their contexts and uses, and regarding them only as sets of propositions with truth values attached to them [80]. The study of real arguments was confined in the realm of rhetoric, which became increasingly a study of the presentation of discourse.

This formal paradigm provided clear and sharp criteria for determining the validity of inferences. However, only a very limited number of natural arguments can be analyzed and assessed according to formal criteria, which address representations of meaning without taking into account their communicative function and their context of use. Argumentation theory attempted to address this gap. However, the dichotomy between formal logic and rhetoric influenced the way this problem was tackled. The rhetorical perspective [56] provided lists of commonly used arguments, without clear criteria for evaluating them. The logical approach applied the formal syllogistic rules to the evaluation of arguments abstracted from their communicative function, the interlocutors, and their context. The outcome was a description of fallacies that mirrored very imperfectly and partially the acceptable and deceptive uses of arguments in dialogues [19,33]. Walton chose a third path, the dialectical one [17], in which arguments are regarded as communicative events, characterized by distinct contexts, functions and effects [74]. The object of analysis is a "pragmatic" inference $[24,34,38]$, namely an inference triggered by an utterance and not necessary [80]:

Presumptive reasoning, according to the analysis given in this book, is based on pragmatic implicatures drawn out by a hearer on the basis of what a speaker's remarks suggest, as opposed to logical inferences that necessarily follow from what a speaker asserts. Hence presumptive reasoning is more rough-and-ready, more simplistic, and also more subject to defeat (and also error) than the logically tight deductive inferences that have traditionally been studied in formal logic.

However, how to assess inferences that are context-dependent and only plausible? The formal criteria clearly cannot help. A different instrument is needed, namely argumentation schemes.

Argumentation schemes are grounded on two fundamental dialectical pillars. The first is constituted by the maximae propositiones of the dialectical tradition, i.e., the abstract conditional premises that guarantee the step from the premises to a conclusion. The history of dialectics devoted a central place to such maxims, which represented the inferential links that are commonly accepted by everyone and underlie the most common acceptable arguments [39]. The second pillar is dialectical in the sense of dialogical: arguments are used in different types of dialogue, each defined by distinct presumptions, levels, and types of cooperativeness with which the speakers are expected to comply [80]. On this perspective, the vague Gricean maxim of cooperativeness is specified by defining the verbal activities on what the interlocutors are cooperating, which results in different presumptions. The type of normativity 
on which argumentation schemes are based is the result of these two dialectical dimensions. The correct use of schemes provides a prima facie reason for accepting a conclusion: as they are grounded on the premises that are commonly accepted by everyone (endoxa), they can be considered as acceptable in lack of contrary reasons. This notion of "validity" is clearly not truth conditional. It is rather procedural, as an argument does not establish a "truth" (whatever it means), but rather a defeasible presumption in favor of a conclusion, which shifts the burden of disproving it onto the interlocutor [80]. The critical questions represent the defeasibility conditions under which the burden shifts back onto the speaker [80].

The normativity of argumentation schemes is thus twofold. They set the constraints of the presumptions used to address a difference in a specific dialogical context [79] by indicating the conditions under which a claim can be presumptively accepted, and the defeasibility conditions that shift the burden back. Argumentation schemes are thus "filters," or dialectical instruments, for determining and regulating how presumptions are set in a particular dialogue [80]. They are constituted by a set of premises leading to a conclusion, where the premises and the conclusion are forms of dialectical statements [32], and a set of critical questions establishing the conditions under which the conclusion can be provisionally accepted. When a critical question is asked or is proven to apply to the argument, the burden of providing evidence or proving that the condition applies shifts back onto the speaker. In this sense, argumentation schemes presuppose a dialogical context (determining their acceptable use for advancing a conclusion presumptively acceptable) and shape it by providing for the future dialogical conditions under which the conclusion can be presumed to be acceptable.

Argumentation schemes are dialectical instruments, and for this reason using a different framework for analyzing or classifying them is highly problematic. For example, we consider the following argumentation scheme from practical reasoning [80,91]:

\begin{tabular}{ll}
\hline PREMISE 1 & Agent $A$ has a goal $G$. \\
PREMISE 2 & Carrying out this action $B_{i}$ is the most acceptable (necessary/sufficient) means to realize $G$. \\
CONCLUSION & Therefore, $A$ should bring about action $B_{i}$. \\
\hline
\end{tabular}

This scheme provides a presumptive reason to a specific practical commitment (performing $B_{i}$ ), and its use is assessed based on the following critical questions:

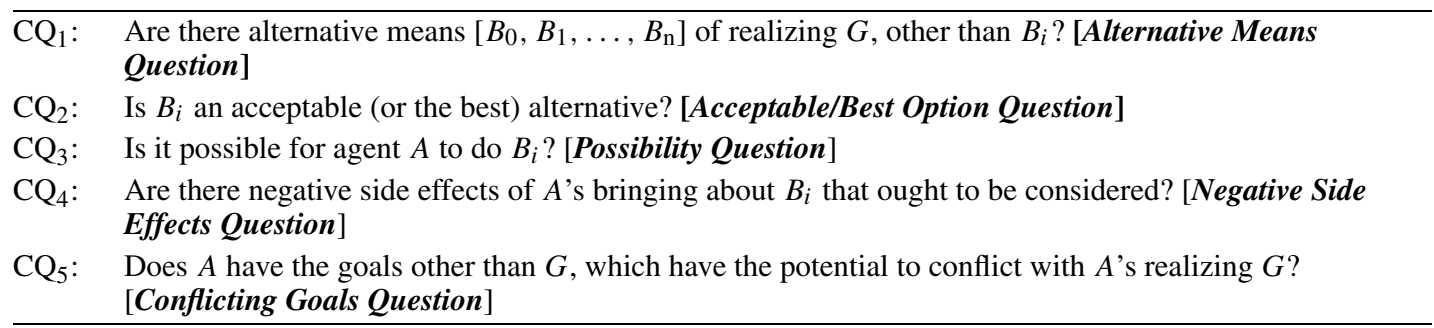

Clearly, it is possible to try to find a "formal" logic in it, and claim that this scheme is abductive, but this account does not distinguish it from other schemes that follow this abductive form. It is possible to look for the semantic relationship between premises and conclusion, and find an underlying cause-effect relationship, but this would not correctly depict the scheme, and would not distinguish it from others. Schemes involve a reasoning, as they represent inferences, and they are grounded on commonly ac- 


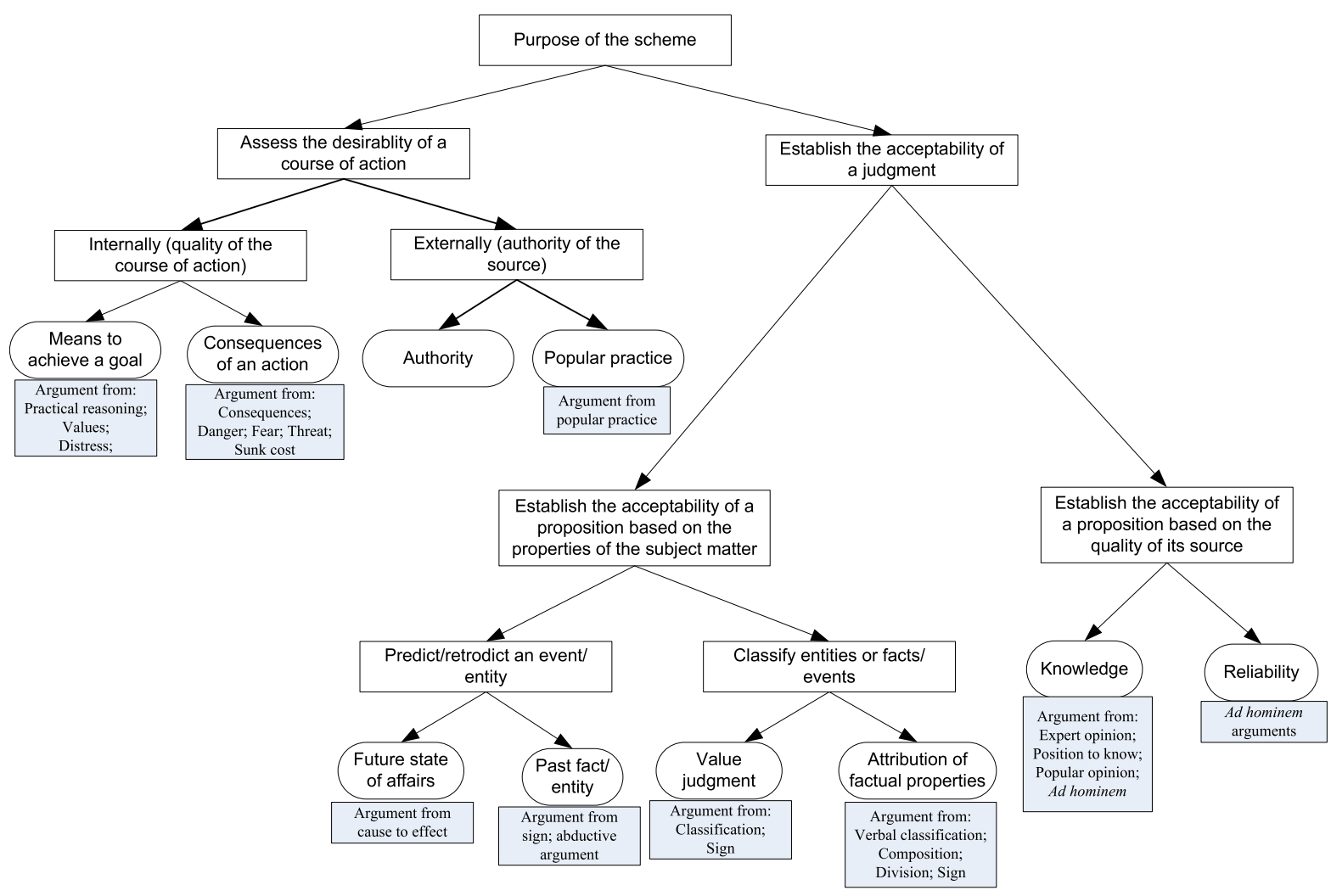

Fig. 1. Pragmatic classification of schemes.

cepted semantic or "rhetorical" relations between the statements that form them. Schemes involve both dimensions [48], but they are not only rhetorical and formal relations. Walton immediately acknowledged the complexity of organizing the schemes, and suggested only clusters of arguments, where some basic schemes were distinguished from their specifications [80]. According to this criterion, an argument like the argument from consequences is considered as basic, while the slippery slope argument or the argument from fear appeal are specifications thereof - the former is a chain of consequences, while the latter is a specific type of consequence. In the following years, argumentation schemes have been classified according to the type of conditional premise, namely identifying some abstract rhetorical relations under which other schemes can fall [91]. This criterion was useful for instructional purposes but lacked clear identification criteria and a tree structure that could allow the choice of a scheme. The most recent developments instead try to focus on the pragmatic nature of the schemes, namely what the argument instantiating the scheme is used for. By changing the perspective on the scheme, it was possible to provide dichotomic criteria that could be used to narrow down and identify the scheme. The selection criteria resulted in the above classification tree (Fig. 1) [47-49].

Argumentation schemes are thus the modern descendants of the dialectical topics. They are essentially pragmatic, and their use consists in the analysis and production of natural arguments - i.e., dialogical events. Clearly, their nature is at the same time an advantage and a limitation for their computational applications, which results in a challenge that has been taken up by many researchers, opening a growing 
stream of research $[8,57,77]$. In the following section, an overview of the different directions of this area of investigation will be provided, reviewing the literature that has addressed the relationship between argumentation schemes and AI.

\section{Argumentation schemes in artificial intelligence}

This short literature review is not intended to be exhaustive - it is only intended to provide an outline of the field by taking into account indexed publications. Scopus was used to search for studies using the keywords 'argument* scheme,' 'artificial intelligence,' and 'mining.' No date limitation was chosen. The initial search returned 128 papers, excluding duplicates. A second screening focused on 2 main criteria: 1) the paper needs to concern the argumentation schemes as developed within Walton's framework, and 2) the paper needs to provide an original account or contribution (excluding literature reviews or commentaries) within the field of artificial intelligence. Applying this criterion, 26 works were excluded, of which seven were grounded on models of schemes different from Walton's. The references of the screened articles were also thoroughly examined to include the foundational works on which they were based that complied with the aforementioned criteria. As a result, nine studies were added. The complete screening process resulted in a total of 111 studies published from 2000 to 2021, thus covering a 22year period of research. The year 2000 corresponds to the year in which the Bonskeid Symposium on Argument and Computation took place [8], where philosophers (including Douglas Walton) and computer scientists met, developing ideas later published in a volume [60].

The corpus of the collected and screened studies was analyzed based on two basic criteria: their purpose and their contribution to AI. The first criterion captures the nature of the works, which can be classified in two macro-areas, namely theoretical works, and computational applications. The first category includes studies that provide classifications, validations, formalizations, or quasi-formalizations of the schemes aimed at making their use in protocols or computer applications possible. Theoretical studies start from a computational problem to develop the schemes, but without providing a formalization to be directly used in AI. In contrast, the category of "computational applications" includes studies that use directly or indirectly the schemes in tools or protocols, which can be divided in three categories: 1) works that provide the formalization for applications, instruments, or protocols (theoretical); 2) works that propose and develop an application or protocol; and 3) studies devoted to the empirical testing or validation of an application or an instrument. The line between "theory" and "practice" is often blurred, as formalizations can be later applied in programs, and programs require specific formalizations - namely a theoretical side. The line was drawn considering the formal framework used, distinguishing the formalizations based on the semantics used in AI (such as Dungean semantics) from the more philosophical ones.

The second criterion concerns the relevance for the area of Artificial Intelligence. Four broad categories were identified, namely argument mining, argument analysis and representation, argument support, and multiagent systems. These areas represent the most important types of research trends in the field of argument and computation. The results of this analysis are reported in Tables $1 ; 2$.

A third type of analysis was further applied, namely the social concern of the studies. This analytical level is intended to grasp the relationship between the computational use or development of the schemes and the area to which such work can contribute. Since this criterion does not characterize all the works scrutinized, only a quantitative outlook is provided. Five extremely broad social sectors were distin- 
Table 1

Theoretical areas of research on argumentation schemes and AI

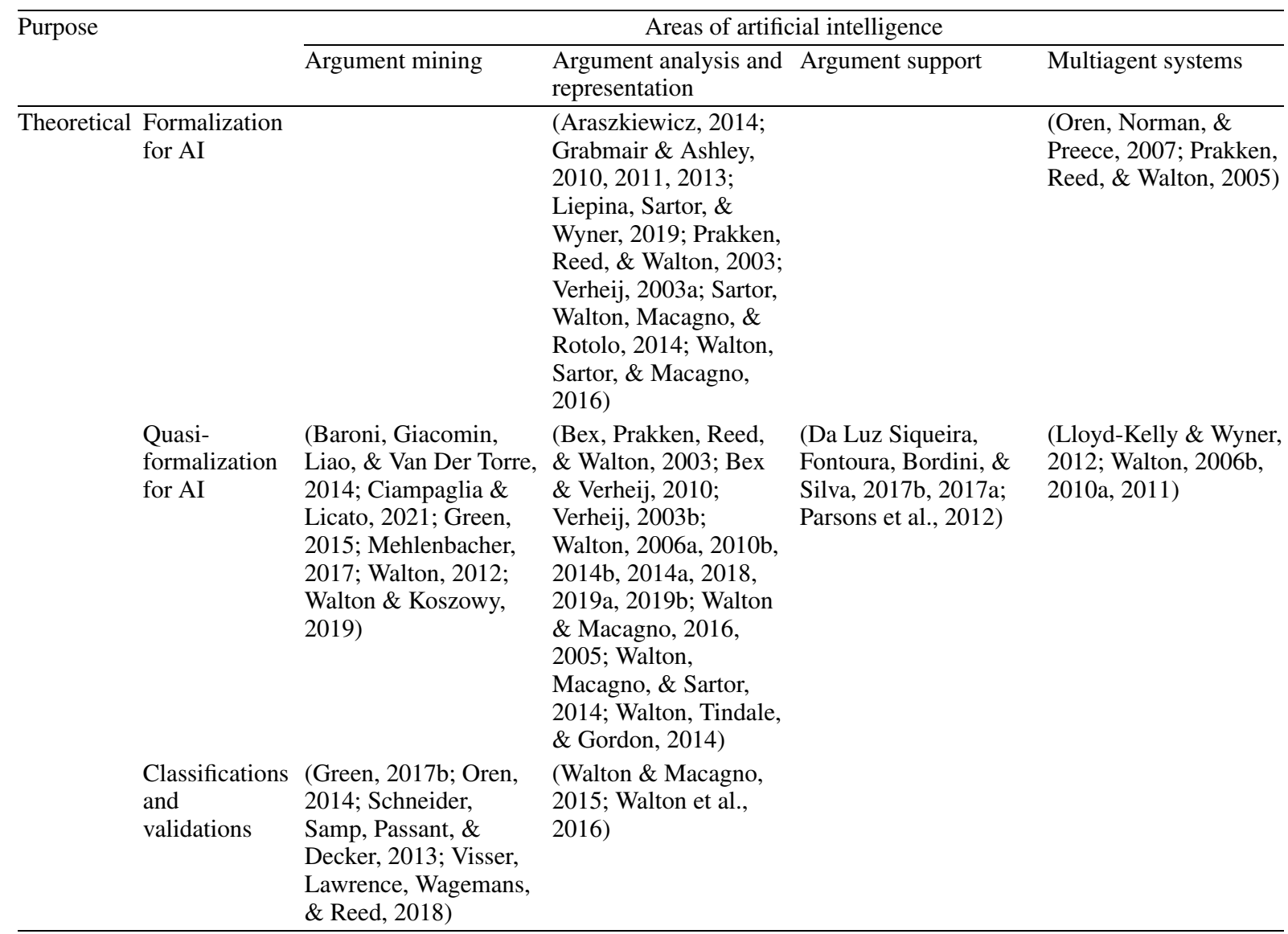

guished, namely law (distinguishing between case law and interpretation), policy making (both at the political and economic level), economics, health, and education, plus a general category of "other social concerns" that includes studies that do not fall into the previous groups. At the level of subject matter, 68 studies addressed an area of social practice, contributing to a specific social challenge. The most representative area is the law (30 works), and in particular case law (25). Policy making (9), healthcare (7), economics (5) and education (3) are also addressed in the corpus, while 14 studies provided research that does not concern any specific field within the social sciences.

Overall, research on argumentation schemes in AI is mostly devoted to the formalization of schemes or their inclusion in formal systems for computing implementations (50 works, amount to $45 \%$ of the total). The theoretical, quasi-formal approaches are 27 (of which 5 are not directly relevant to AI). 28 studies propose programs, almost equally distributed in the areas of argument mining (8), argument analysis and representation (9), support to the production of arguments (7), and multiagent systems (6). The empirical works are 6 in total, 3 aimed at validating the theoretical frameworks for the annotation of schemes, and 3 providing the outcomes of AI applications of schemes. 
Table 2

Areas of research on argumentation schemes and AI: AI applications

\begin{tabular}{|c|c|c|c|c|c|}
\hline \multirow[t]{2}{*}{ Purpose } & & \multicolumn{4}{|c|}{ Areas of artificial intelligence } \\
\hline & & Argument mining & $\begin{array}{l}\text { Argument analysis } \\
\text { and representation }\end{array}$ & Argument support & Multiagent systems \\
\hline \multirow[t]{3}{*}{$\begin{array}{l}\text { Computational } \\
\text { application }\end{array}$} & $\begin{array}{l}\text { Theoretical } \\
\text { contribution }\end{array}$ & $\begin{array}{l}\text { (Green, 2016, 2017a, } \\
\text { 2018; D Liga \& } \\
\text { Palmirani, 2020a) }\end{array}$ & $\begin{array}{l}\text { (Atkinson, } \\
\text { Bench-Capon, \& } \\
\text { McBurney, 2005; } \\
\text { Bench-Capon, 2012; } \\
\text { Bex, Bench-Capon, \& } \\
\text { Atkinson, 2008; } \\
\text { Gordon \& Walton, } \\
\text { 2009; Green, 2011; } \\
\text { Oren, 2014; Prakken, } \\
\text { 2012b, 2012a; } \\
\text { Pulfrey-Taylor, } \\
\text { Henthorn, Atkinson, } \\
\text { Wyner, \& } \\
\text { Bench-Capon, 2011; } \\
\text { Wyner, Bench-Capon, } \\
\text { \& Atkinson, 2011) }\end{array}$ & $\begin{array}{l}\text { (Belahcene, } \\
\text { Labreuche, Maudet, } \\
\text { Mousseau, \& } \\
\text { Ouerdane, 2017; Bex } \\
\text { et al., 2008; Da Luz } \\
\text { Siqueira et al., 2017b; } \\
\text { Dunne \& } \\
\text { Bench-Capon, 2004; } \\
\text { Gordon \& Walton, } \\
\text { 2009; Green, 2011; } \\
\text { Müller, Hunter, \& } \\
\text { Taylor, 2013; } \\
\text { Ouerdane, } \\
\text { Dimopoulos, Liapis, } \\
\text { \& Moraitis, 2011; } \\
\text { Ouerdane, Maudet, \& } \\
\text { Tsoukias, 2008; R. } \\
\text { Thomas, Masthoff, \& } \\
\text { Oren, 2019; Walton, } \\
\text { Oliveira, Satoh, \& } \\
\text { Mebane, 2021) }\end{array}$ & $\begin{array}{l}\text { (Atkinson \& } \\
\text { Bench-Capon, 2007; } \\
\text { Bench-Capon \& } \\
\text { Prakken, 2010; } \\
\text { Bench-Capon, } \\
\text { Prakken, \& Visser, } \\
\text { 2011; Dunne \& } \\
\text { Bench-Capon, 2004; } \\
\text { Maio \& Silva, 2012; } \\
\text { Medellin-Gasque, } \\
\text { Atkinson, \& } \\
\text { Bench-Capon, 2012; } \\
\text { Medellin-Gasque, } \\
\text { Atkinson, McBurney, } \\
\text { \& Bench-Capon, } \\
\text { 2012; Shams, De Vos, } \\
\text { Oren, Padget, \& } \\
\text { Satoh, 2015; Toniolo, } \\
\text { Norman, \& Sycara, } \\
\text { 2011; Vassiliades et } \\
\text { al., 2020; Wardeh, } \\
\text { Bench-Capon, \& } \\
\text { Coenen, 2007; Wells, } \\
\text { 2018) }\end{array}$ \\
\hline & $\begin{array}{l}\text { Instrument } \\
\text { (protocol or } \\
\text { program) }\end{array}$ & $\begin{array}{l}\text { (Cabrio, Tonelli, \& } \\
\text { Villata, 2013; Green } \\
\text { \& Crotts, 2021; } \\
\text { Lawrence \& Reed, } \\
\text { 2015; D Liga \& } \\
\text { Palmirani, 2019a, } \\
\text { 2019b, 2020b; Davide } \\
\text { Liga, 2019; Wei Feng } \\
\text { \& Hirst, 2011) }\end{array}$ & $\begin{array}{l}\text { (Gordon, 2011; } \\
\text { Green, 2013; Green, } \\
\text { Branon, \& Roosje, } \\
\text { 2019; Lenz, Ollinger, } \\
\text { Sahitaj, \& Bergmann, } \\
\text { 2019; Rahwan, } \\
\text { Zablith, \& Reed, } \\
\text { 2007; Walton \& } \\
\text { Gordon, 2012; } \\
\text { Wyner, 2014; Y } \\
\text { Zagorulko, Domanov, } \\
\text { Sery, Sidorova, \& } \\
\text { Borovikova, 2020; } \\
\text { Yury Zagorulko, } \\
\text { Garanina, Sery, \& } \\
\text { Domanov, 2019) }\end{array}$ & $\begin{array}{l}\text { (Gordon, 2011; } \\
\text { Green, 2013; Janjua, } \\
\text { Hussain, \& Hussain, } \\
\text { 2013; R. J. Thomas, } \\
\text { Collinson, \& } \\
\text { Masthoff, 2018; R. } \\
\text { Thomas, Oren, \& } \\
\text { Masthoff, 2018; } \\
\text { Tolchinsky, Modgil, } \\
\text { \& Cortés, 2006; } \\
\text { Verheij, 2003a) }\end{array}$ & $\begin{array}{l}\text { (Atkinson, } \\
\text { Bench-Capon, } \\
\text { Cartwright, \& Wyner, } \\
\text { 2011; Costa et al., } \\
\text { 2017; Panisson \& } \\
\text { Bordini, 2018; } \\
\text { Rahwan \& } \\
\text { Banihashemi, 2008; } \\
\text { Rahwan et al., 2007; } \\
\text { Wyner, Atkinson, \& } \\
\text { Bench-Capon, 2012) }\end{array}$ \\
\hline & $\begin{array}{l}\text { Empirical / } \\
\text { testing }\end{array}$ & $\begin{array}{l}\text { (Dumani et al., 2021; } \\
\text { Kononenko, } \\
\text { Sidorova, \& } \\
\text { Akhmadeeva, 2020) }\end{array}$ & (Dumani et al., 2021) & & $\begin{array}{l}\text { (Toniolo, Norman, \& } \\
\text { Sycara, 2012) }\end{array}$ \\
\hline
\end{tabular}

\section{Trends of research}

The research on argumentation schemes and Artificial intelligence can be mapped in some broad trends, which capture at the same time the most promising areas and the open challenges. Below are outlined some of these trends based on the aforementioned results of our literature review. 


\subsection{Theoretical developments}

Argumentation schemes are at the same time a resource and a challenge for the world of Artificial Intelligence. Their potential lies in the accuracy and specificity of analysis that can be performed by using them: schemes allow the detection of the types and subtypes of arguments expressed in a text (or to be produced) without resorting to an artificial semantic system [12]. However, the drawback of this precision is the complexity of its use, which emerges even at the level of manual annotation and is due to two types of issues, one theoretical and the other practical. From a theoretical point of view, the number of schemes is too high to guarantee agreement between coders, and the lack of classification criteria or decision trees has led to difficulties in their use. Attempts to test the reliability of this instrument have achieved results ranging from fair [23,65] to satisfactory results by reducing the number of schemes to few categories [29], or involving expert annotators [78]. From a practical point of view, the use of schemes need to face the complexity of real texts, which often involve chained arguments or can be interpreted in different ways [78]. To address these problems, schemes have been specified by identifying their distinguishing features [84-86,88], and organized in binary categories allowing theory-driven decision trees $[89,92]$. The most promising theoretical developments are either directed to the simplification of the system of schemes, or the formalization of their manifestation in specific contexts [65]. The introduction of new schemes to capture specific types of argument characterizing a type of text $[46,50,55,81]$ can increase reliability, but reduce the applicability of the instrument.

The theoretical development of schemes is crucial for the creation of "gold standard" corpora, namely annotated databases that can be used for training automatic classifiers [23]. Few databases of arguments are publicly available [61], and the training is often performed on private datasets whose reliability and coherence with argumentation schemes theory is not specified. Very rarely do annotated corpora include detailed information about the coding procedure and accurate reliability measures [23].

\subsection{Argument mining}

Perhaps the most ambitious project in the field of argumentation and AI is the automatic detection of the types of arguments [51,66] (23 studies are devoted to this endeavor). Argumentation schemes provide categories of arguments, together with criteria for recognizing them; however, a direct matching between the stereotypical, quasi-formal representations of premises and conclusions and natural arguments is impossible. Natural arguments are characterized by an implicit dimension and the possibility of being expressed in many different ways - in sum, they are incomplete and based on an extremely complex ontology [45]. The classical methods for argumentation scheme detection are based on textual features, normally keywords [30,41]. This approach has provided relevant results; however, the success is normally limited to specific conditions - a simplified database (a corpus of pre-identified arguments with explicit conclusions [95]), or specific classification conditions (pairwise identifications). However, the combination between the distinct textual (superficial) features, such as discourse indicators, scheme structure, or topical similarity, had led to satisfactory outcomes also when used on databases not previously analyzed and simplified [42]. A different strategy consists in applying automatic classifiers to databases previously interpreted - for example, with discourse relations manually identified and classified [18] - or conversely specifying the schemes to a specific topic and thus narrowing the gap between the ontology of the scheme and the one of the text [30]. A third approach is focused on the argument pipeline that can eventually lead to or facilitate argumentation schemes identification. For example, argument elements (evidence, oppositions, etc.) have been classified in macro-categories (anecdote, study, 
statistics, etc. or judgment, moral judgment, slippery slope, etc.) using tree kernels (measures of similarity between syntactic-semantic structures occurring in texts), making it possible to get closer to argument detection $[43,44]$.

\subsection{Argument analysis and representation}

One of the most productive trends of research involving argumentation schemes is argument representation and analysis, especially in the field of law [26,87]. The use of argumentation schemes in the representation of arguments pursues two goals. The first is to provide an evaluation of a complex argumentation [10] - in addition to its visualization and analysis - either through dedicated software systems or formal argumentation frameworks $[13,15]$. The second is to develop a World Wide Argument Web (WWAW), namely a structured web of interconnected arguments posted online, which can be searched by topic or by structure [59].

This latter aim characterizes the efforts devoted to developing tools such as Araucaria, OVA, and ArgDF, in addition to a "common language," namely a shared ontology for representing argumentation, that can be used by different tools and systems (AIF). Among the most known software visualization tools, Araucaria [62] was the first that integrated argumentation schemes. Araucaria allows users to represent the manually identified or reconstructed premises and conclusions of an argument as a graph, associating the link leading from the premise-nodes to the conclusion node with an argumentation scheme. Moreover, it allows the identification of the speaker and thus can represent actual dialogues. The Online Visualization of Argument (OVA) and ArgDF are based on a common ontology - the Argumentation Interchange Format (AIF) - that provides a common, formalized perspective on argumentation, facilitating data exchange between different tools for argument visualization. OVA [36] is an interface for the analysis of arguments online, which allows the specification not only of the argumentation scheme, but also of the role of each premise (for example, the expertise premise in arguments from authority, etc.), and the strength of the argument, assessed through critical questions. The System for Authoring and Navigating Arguments (ArgDF) [58,59] is a Semantic Web-based system that allows the creation, visualization, and manipulation of arguments (also by attacking arguments and creating new argumentation schemes), in addition to semantically querying semantically annotated arguments on the Web. The last well-known system of argument representation, developed principally for case-law, is Carneades $[25,27,87]$. Carneades is a formal model of argumentation on which a software visualization and evaluation system is based, based on a specific abstract argumentation framework. Arguments are represented both as products and as parts of argumentation processes - i.e., as related to a network of arguments for or against a certain conclusion. The original aspect of Carneades is the attribution to each statement of a standard of proof, which allows the prediction of the burden shifting.

Carneades illustrates the other fundamental goal of using schemes in formal and visual representations of arguments, namely assessing the outcome of an argumentation process. In Carneades, it is possible to attribute to each premise a numerical weight, corresponding to conditional probabilities, and distinguish its status (accepted, defeated, attacked) [28]. The schemes and the relationships between arguments allow the calculation of the possible acceptability of a conclusion. Also other argument representation systems include strategies for evaluating arguments, based on their structure (argumentation scheme) and the relations with other arguments, attacks, and counterarguments [97,98]. Critical questions are used to assess the strength of individual arguments and the attack relationships between arguments can connect arguments in networks, distinguishing the conclusions based on the attacks received. A further step was provided by the introduction of preference rules among schemes [16,63,92], which allow predicting the relative superiority of an argument over another. 


\subsection{Argument assistance and decision support}

Argumentation schemes are also used to support the "invention" of arguments by users in argument assistance programs [75,76]. Argument analysis and visualization tools are designed for annotating texts and reconstructing the relationships between arguments. A different approach is provided by systems that help users construct, evaluate, and challenge their own arguments. An example is AVIZE (Argument Visualization and Evaluation) [31], which supports users in the evaluation of the available evidence and its use in arguments, represented in forms of diagrams. Argumentation schemes guide the process of invention by providing the conditions for an inference scheme to apply and support the argument evaluation by showing the critical aspects thereof. Similar systems are structured as templates, in which the user is guided in the choice of an argumentation scheme, which s/he then selects; this choice leads to a set of questions, whose answers are then organized and structured in an semi-automatically generated argumentative message [67-69]. Such templates can be adjusted to specific fields or disciplines by creating specific, ad hoc schemes. By following specific argument templates, users produce standardized arguments, making it possible for the discussants to detect, assess, and reuse arguments [20,21].

A different type of systems is designed to support the decision-making processes by constructing, representing, and evaluating the reasons in favor or against a specific decision [52,53]. Argumentation schemes are used for assessing and integrating the new information and the new reasons: critical questions guide the user in accepting or excluding reasons or information, updating the system representing the overall structure of the pro and con arguments [37]. The dialectical nature of argumentation schemes has been used for eliciting from doctors the information necessary for deciding about the viability of organs for transplantation [71], combining different arguments and information on a specific medical issues, supporting the decision making in treatment issues [90], and collecting, comparing, and evaluating arguments in favor or against policies [9].

\subsection{Multiagent systems}

Decision-support systems represent a specific development of a much broader research area, consisting in the development of computerized dialogical processes between different agents (machine-machine or machine-human interactions, see [96]). The most important trend within this area is decision-making, represented using the argumentation scheme from practical reasoning [6,11,14], and dialogue norms based on the deliberation dialogue [94]. This vast area can be mapped by considering three types of works: 1) theoretical developments of dialogue models, setting out the rules of an interaction; 2) developments of formal or computational argumentation frameworks regulating interactions; and 3) empirical testing of the effectiveness of a multiagent system.

Walton greatly contributed to the theoretical developments of dialogue models, outlining rules and procedures governing different types of dialogues (see for instance [83]) or specifying the relationship between types of dialogues, moves, and argumentation schemes [82,93,94]. The formal and computational developments represent the major trend. Atkinson and colleagues outlined a formal system in which the distinction between the types of premises allows the detection of the values underlying a practical conclusion, while the critical questions and the preference ordering allows a system to automatically compare arguments, recommending a proposal [7,9]. Dialogical protocols have been also combined by Oren and colleagues with argumentation schemes and evidence in an argumentation framework in which agents exchange arguments that are then evaluated considering the available evidence, resulting in a conclusion that corresponds to what the interlocutors should agree upon. A distinct approach is constituted 
by attempts to include in the argumentation framework the contextual, organizational circumstances that define not only the agents' roles, but also the types of arguments used by the agents [54].

Finally, empirical works are aimed at determining statistically the advantages of a specific system. For example, the use of argumentation schemes in multiagent systems has been proven also empirically to affect the quality of deliberation. Toniolo and colleagues [72,73] showed how a model of deliberation dialogue based on argumentation schemes leads to a higher resolution of conflicts between the agents, resulting in more collaborative plans.

\section{This special issue}

This Special Issue provides a twofold contribution to this complex area of research. On the one hand, it addresses some fundamental challenges, both theoretical and applicative, by advancing new proposals. On the other hand, it maps the uses of argumentation schemes in artificial intelligence from the perspective of three broad fields of application, namely biology, international relations, and law.

Mood and force in defeasible arguments raises a theoretical question that exceeds the boundaries of linguistics and philosophy of language to become a very practical issue for any use of argumentation schemes: how to interpret the force of utterances in arguments, and even more importantly, what utterances are for in a dialogue? How can we claim that an utterance is an assertion, a command, or a question, and has been produced for a specific dialogical goal? These questions point out one of the crucial limitations of AI when pursuing the goal of coping with real texts: interpretation is a defeasible endeavor and linguistic indicators - such as mood - can provide only tentative, presumptive conclusions. Quandt and Licato distinguish not only force from its linguistic manifestation, i.e., grammatical mood, but also from the dialogical acts that are performed through an utterance. The defeasibility of interpretation is addressed by the use of argumentation schemes. The force setters provide a defeasible indication of the possible use of an utterance in an argument of a certain type, represented by an argumentation scheme; however, this hypothesis needs to be tested by critical questions, which assume an intent and verify its relevance to the dialogue (whether the interpretation would lead to a relevant move) and the commitments that the speaker would take on.

Two papers outline AI applications of argumentation schemes, contributing to two distinct streams of research, namely argument support systems and multiagent systems. Argumentation schemes for clinical decision support concerns the issue of patient-doctor communication and decision-making. In clinical practice, especially in multi-morbidity situations, clinical decision support systems can help collect, compare, interpret the data from different sources and provide justifications and reasons for treatment options. The core of this proposal lies in the use of schemes, which are a bridge between human dialogues and formal systems. However, to be used, schemes and critical questions need to be adapted to the specific domain of practice, so that they can represent the actual, most common types of specific arguments used in a given medical context. The authors provide such schemes based on a General Practitioner's experience and develop a decision support system in which clinical data are combined with medical guidelines to instantiate an argumentation scheme. The argument resulting from this operation is combined within an argumentation framework with other conflicting or supporting arguments, assessed through critical questions and argument preferences, leading to a recommendation.

In A computational model of argumentation schemes for multi-agent systems, Panisson, McBurney and Bordini present a novel argumentation framework in which argumentation schemes are used for multi-agent communication. In particular, their formalism allows agents to use the schemes, detect the 
argumentation schemes expressed by the other agents' arguments, and request clarification in case it is not possible to identify a scheme with certainty. Moreover, this system allows addressing deep disagreement by allowing sub-dialogues on the specific instantiation of a scheme, using critical questions to bring to light and evaluate the implicit premises used. This framework is evaluated by implementing it in the Jason multi-agent platform, calculating the time needed for constructing an argument using a scheme considering different variables, such as the critical questions and nested arguments.

The last two papers provide a focused review of the AI literature on the use of the schemes in specific domains. Green, with her paper on Argumentation schemes: From genetics to international relations to environmental science policy to AI ethics shows the research path followed to use the schemes in computational applications for representing real arguments and supporting the production of arguments. As the author points out, the passage from texts to the formalizations needed for AI is bridged by schemes, which needed to be adapted to different genres and subject domains. The development of annotation schemes and more importantly annotation corpora is necessary for machine training, while argument representation can be used not only for analytical purposes but also for argument support systems. Through an overview of her projects, Green offers a critical perspective on how schemes can be used in specific domains, and the future steps.

The last paper Argumentation schemes in AI and law concerns the subject domain that has stimulated more research in AI and argumentation schemes, namely the law. Atkinson and Bench-Capon illustrate the different trends of research, distinguishing the logical and dialogical nature of schemes. Presenting the background of legal research before the use of schemes, the authors show how this tool has contributed to representing different types of arguments used in legal discussions or statutory interpretation, modelling the creation of arguments, evaluating legal reasons through critical questions, and developing dialogical protocols that define sequences of moves based on the type of argument and the corresponding critical questions.

As this short literature review shows and the contributions to this special issue testify, argumentation schemes are a methodological instrument that finds more and more computational applications and is applied in some of the most ambitious areas of research in AI. Douglas Walton understood the importance of the schemes both at a philosophical and computational level, and enthusiastically dialogued with the world of AI, which he always acknowledged as inherently related with philosophy. The outcomes of this research, which inevitably is partially, incompletely, and approximately presented in this introduction, are clear evidence of how Doug contributed to shaping the area of argument and computation, revealing the philosophical dimension of AI, and the need of philosophical tools such as the schemes for chasing some computational dreams.

\section{Acknowledgement}

I would like to thank the Fundação para a Ciência e a Tecnologia for the research grants PTDC/FERFIL/28278/2017 and UIDB/00183/2020 and the CHIST-ERA Programme for the research grant CHISTERA/0002/2019.

\section{References}

[1] P. Abaelardus, Dialectica, Van Gorcum, Assen, Netherlands, 1970. 
[2] Aristotle, Topics, in: The Complete Works of Aristotle, Vol. I, J. Barnes, ed., Princeton University Press, Princeton, NJ, 1991.

[3] Aristotle, Rhetoric, in: The Complete Works of Aristotle, Vol. II, J. Barnes, ed., Princeton University Press, Princeton, NJ, 1991.

[4] Aristotle, Sophistical refutations, in: The Complete Works of Aristotle, Vol. I, J. Barnes, ed., Princeton University Press, Princeton, NJ, 1991.

[5] A. Arnauld and P. Nicole, Logic or the Art of Thinking, Cambridge University Press, Cambridge, UK, 1996.

[6] K. Atkinson and T. Bench-Capon, Practical reasoning as presumptive argumentation using action based alternating transition systems, Artif Intell 171 (2007), 855-874. doi:10.1016/j.artint.2007.04.009.

[7] K. Atkinson and T. Bench-Capon, Action-based alternating transition systems for arguments about action, in: AAAI'07: Proceedings of the 22nd National Conference on Artificial Intelligence, A. Cohn, ed., AAAI Press, Palo Alto, CA, 2007, pp. 24-29.

[8] K. Atkinson, T. Bench-Capon, F. Bex et al., In memoriam Douglas N. Walton: The influence of Doug Walton on AI and law, Artif Intell Law 28 (2020), 281-326. doi:10.1007/s10506-020-09272-2.

[9] K. Atkinson, T. Bench-Capon, D. Cartwright et al., Semantic models for policy deliberation, in: Proceedings of the International Conference on Artificial Intelligence and Law, K. Ashley and T. van Engers, eds, ACM, New York, NY, 2011, pp. 81-90.

[10] K. Atkinson, T. Bench-Capon and P. McBurney, Arguing about cases as practical reasoning, in: Proceedings of the 10th International Conference on Artificial Intelligence and Law, G. Sartor, ed., ACM Press, New York, NY, 2005, pp. 35-44. doi:10.1145/1165485.1165492.

[11] K. Atkinson, T. Bench-Capon and P. McBurney, Computational representation of practical argument, Synthese 152 (2006), 157-206. doi:10.1007/s11229-005-3488-2.

[12] P. Baroni, M. Giacomin, B. Liao et al., Encompassing uncertainty in argumentation schemes, in: CEUR Workshop Proceedings, New York, NY, 2014.

[13] T. Bench-Capon, Representing Popov v Hayashi with dimensions and factors, Artif Intell Law 20 (2012), 15-35. doi:10. 1007/s10506-012-9118-7.

[14] T. Bench-Capon and H. Prakken, A lightweight formal model of two-phase democratic deliberation, Epub ahead of print, 2010. doi:10.3233/978-1-60750-682-9-27.

[15] F. Bex, T. Bench-Capon and K. Atkinson, Did he jump or was he pushed? Abductive practical reasoning, in: Frontiers in Artificial Intelligence and Applications, E. Francesconi, G. Sartor and D. Tiscornia, eds, IOS Press, Amsterdam, Netherlands, 2008, pp. 138-149.

[16] F. Bex and C. Reed, Schemes of inference, conflict, and preference in a computational model of argument, Stud Logic, Gramm Rhetor 23 (2011), 39-58.

[17] J.A. Blair, R.H. Johnson, A. Blair et al., Argumentation as dialectical, Argumentation 1 (1987), 41-56. doi:10.1007/ BF00127118.

[18] E. Cabrio, S. Tonelli and S. Villata, A natural language account for argumentation schemes, in: AI*IA 2013: Advances in Artificial Intelligence, M. Baldoni, C. Baroglio, G. Boella et al., eds, Springer, Cham, Switzerland, 2013, pp. $181-192$. doi:10.1007/978-3-319-03524-6_16.

[19] I. Copi and C. Cohen, Introduction to Logic, 12th edn, Prentice-Hal, Upper Saddle River, 2005.

[20] D. Da Luz Siqueira, L.M. Fontoura, R.H. Bordini et al., Argumentation schemes for collaborative debate of requirement risks in software projects, Int J Softw Eng Knowl Eng 27 (2017), 1613-1635. doi:10.1142/S0218194017400150.

[21] D. Da Luz Siqueira, L.M. Fontoura, R.H. Bordini et al., A knowledge engineering process for the development of argumentation schemes for risk management in software projects, in: Proceedings of the International Conference on Software Engineering and Knowledge Engineering, SEKE, Curran, New York, NY, pp. 36-41.

[22] W. De Pater, Les Topiques d'Aristote et la dialectique platonicienne, Éditions de St. Paul, Fribourg, 1965.

[23] L. Dumani, M. Biertz, A. Witry et al., The ReCAP corpus: A corpus of complex argument graphs on German education politics, in: Proceedings - 2021 IEEE 15th International Conference on Semantic Computing, ICSC 2021, IEEE Computer Society, Washington, DC, 2021, pp. 248-255.

[24] M.L. Geis and A.M. Zwicky, On invited inferences, Linguist Inq 2 (1971), 561-566.

[25] T. Gordon, An overview of the Carneades argumentation support system, in: Dialectics, Dialogue and Argumentation. An Examination of Douglas Walton's Theories of Reasoning and Argument, C. Reed and C. Tindale, eds, College Publications, London, UK, 2010, pp. 145-156.

[26] T. Gordon, Analyzing open source license compatibility issues with Carneades, in: ICAIL'11: Proceedings of the 13th International Conference on Artificial Intelligence and Law, K. Ashley and T. van Engers, eds, ACM, New York, NY, pp. 51-55.

[27] T. Gordon, H. Prakken and D. Walton, The Carneades model of argument and burden of proof, Artif Intell 171 (2007), 875-896. doi:10.1016/j.artint.2007.04.010. 
[28] T. Gordon and D. Walton, The Carneades argumentation framework: Using presumptions and exceptions to model critical questions, in: Computational Models of Argument, P. Dunne and T. Bench-Capon, eds, IOS Press, Amsterdam, Netherlands, 2006, pp. 195-207.

[29] N. Green, Annotating evidence-based argumentation in biomedical text, in: Proceedings - 2015 IEEE International Conference on Bioinformatics and Biomedicine, BIBM 2015, IEEE Computer Society, Washington, DC, 2015, pp. $922-929$. doi:10.1109/BIBM.2015.7359807.

[30] N. Green, Towards mining scientific discourse using argumentation schemes, Argument Comput 9 (2018), 121-135. doi:10.3233/AAC-180038.

[31] N. Green, M. Branon and L. Roosje, Argument schemes and visualization software for critical thinking about international politics, Argument Comput 10 (2019), 41-53. doi:10.3233/AAC-181003.

[32] D.J. Hadgopoulos, Protasis and problema in the "topics", Phronesis (1976), 266-276. doi:10.1163/156852876X00183.

[33] C.L. Hamblin, Fallacies, Methuen, London, UK, 1970.

[34] R. Harris and G. Monaco, Psychology of pragmatic implication: Information processing between the lines, J Exp Psychol Gen 107 (1978), 1-22. doi:10.1037/0096-3445.107.1.1.

[35] A. Hastings, A reformulation of the modes of reasoning in argumentation, $\mathrm{PhD}$ dissertation, Northwestern University, Evanston, IL, 1963.

[36] M. Janier, J. Lawrence and C. Reed, OVA+: An argument analysis interface, in: Computational Models of Argument, S. Parsons, N. Oren, C. Reed et al., eds, IOS Press, Amsterdam, Netherlands, 2014, pp. 463-464.

[37] N.K. Janjua, F.K. Hussain and O.K. Hussain, Semantic information and knowledge integration through argumentative reasoning to support intelligent decision making, Inf Syst Front 15 (2013), 167-192. doi:10.1007/s10796-012-9365-x.

[38] M.K. Johnson, J.D. Bransford and S.K. Solomon, Memory for tacit implications of sentences, J Exp Psychol 98 (1973), 203-205. doi:10.1037/h0034290.

[39] M. Kienpointner, Alltagslogik: Struktur und Funktion von Argumentationsmustern, Fromman-Holzboog, Stuttgart, Germany, 1992.

[40] M. Kienpointner, Towards a typology of argumentative schemes, in: Argumentation: Across the Lines of Discipline, F. van Eemeren, R. Grootendorst, A. Blair et al., eds, Foris, Dordrecht, Netherlands, 1987, pp. 275-287.

[41] J. Lawrence and C. Reed, Argument mining: A survey, Comput Linguist 45 (2020), 765-818. doi:10.1162/coli_a_00364.

[42] J. Lawrence and C. Reed, Argument mining using argumentation scheme structures, in: Computational Models of Argument, P. Baroni, T. Gordon, T. Scheffler et al., eds, IOS Press, Amsterdam, Netherlands, 2016, pp. 379-390.

[43] D. Liga and M. Palmirani, Classifying argumentative stances of opposition using tree kernels, in: ACAI 2019: Proceedings of the 2019 2nd International Conference on Algorithms, Computing and Artificial Intelligence, ACM, New York, NY, 2019, pp. 17-22. doi:10.1145/3377713.3377717.

[44] D. Liga and M. Palmirani, Detecting "slippery slope" and other argumentative stances of opposition using tree kernels in monologic discourse, in: Rules and Reasoning. RuleML+RR 2019, P. Fodor, M. Montali, D. Calvanese et al., eds, Springer, Cham, Switzerland, 2019, pp. 180-189.

[45] D. Liga and M. Palmirani, Argumentation schemes as templates? Combining bottom-up and top-down knowledge representation, in: Proceedings of the 20th Workshop on Computational Models of Natural Argument, F. Grasso, N. Green, J. Schneider et al., eds, CEUR, New York, NY, pp. 51-56.

[46] M. Lloyd-Kelly and A. Wyner, Arguing about emotion, in: Advances in User Modeling. UMAP 2011, L. Ardissono and T. Kuflik, eds, Springer, Berlin, Germany, 2012, pp. 355-367.

[47] F. Macagno, A means-end classification of argumentation schemes, in: Reflections on Theoretical Issues in Argumentation Theory, F. van Eemeren and B. Garssen, eds, Springer, Cham, Switzerland, 2015, pp. 183-201. doi:10.1007/978-3-31921103-9_14.

[48] F. Macagno and D. Walton, Classifying the patterns of natural arguments, Philos Rhetor 48 (2015), 26-53. doi:10.5325/ philrhet.48.1.0026.

[49] F. Macagno, D. Walton and C. Reed, Argumentation schemes. History, classifications, and computational applications, J Appl Logics 4 (2017), 2493-2556.

[50] A.R. Mehlenbacher, Rhetorical figures as argument schemes - the proleptic suite, Argument Comput 8 (2017), $233-252$. doi:10.3233/AAC-170028.

[51] R. Mochales Palau and M.F. Moens, Argumentation mining: The detection, classification and structuring of arguments in text, in: Proceedings of the 12th International Conference on Artificial Intelligence and Law (ICAIL'09), ACM, New York, NY, 2009, pp. 351-352.

[52] W. Ouerdane, Y. Dimopoulos, K. Liapis et al., Towards automating decision aiding through argumentation, J MultiCriteria Decis Anal 18 (2011), 289-309. doi:10.1002/mcda.486.

[53] W. Ouerdane, N. Maudet and A. Tsoukias, Argument schemes and critical questions for decision aiding process, in: Proceedings of the 2008 Conference on Computational Models of Argument: Proceedings of COMMA 2008, P. Besnard, S. Doutre and A. Hunter, eds, IOS Press, Amsterdam, Netherlands, pp. 285-296. 
[54] A.R. Panisson and R.H. Bordini, Argumentation schemes in multi-agent systems: A social perspective, in: Engineering Multi-Agent Systems. EMAS 2017, A. El Fallah-Seghrouchni, A. Ricci and T. Son, eds, Lecture Notes in Computer Science, Springer, Cham, Switzerland, 2018, pp. 92-108. doi:10.1007/978-3-319-91899-0_6.

[55] S. Parsons, K. Atkinson, Z. Li et al., Argument schemes for reasoning about trust, Argument Comput 5 (2014), 160-190. doi:10.1080/19462166.2014.913075.

[56] C. Perelman and L. Olbrechts-Tyteca, The New Rhetoric: A Treatise on Argumentation, University of Notre Dame Press, Notre Dame, IN, 1969.

[57] H. Prakken, AI \& law, logic and argument schemes, Argumentation 19 (2005), 303-320. doi:10.1007/s10503-005-4418-7.

[58] I. Rahwan and B. Banihashemi, Arguments in OWL: A progress report, in: Computational Models of Argument, P. Besnard, S. Doutre and A. Hunter, eds, IOS Press, Amsterdam, Netherlands, 2008, pp. 297-310.

[59] I. Rahwan, F. Zablith and C. Reed, Towards large scale argumentation support on the semantic web, in: AAAI'07: Proceedings of the 22nd National Conference on Artificial Intelligence, A. Cohn, ed., AAAI Press, Palo Alto, CA, 2007, pp. 1446-1451.

[60] C. Reed and T. Norman (eds), Argumentation Machines. New Frontiers in Argument and Computation, Springer, Dordrecht, Netherlands, 2004.

[61] C. Reed, R.M. Palau, G. Rowe et al., Language resources for studying argument, in: Proceedings of the Sixth International Conference on Language Resources and Evaluation (LREC'08), N. Calzolari, K. Choukri, B. Maegaard et al., eds, European Language Resources Association (ELRA), Marrakech, Morocco, 2008, pp. 2613-2618.

[62] C. Reed and G. Rowe, Araucaria: Software for argument analysis, diagramming and representation, Int J Artif Intell Tools 13 (2004), 961-979. doi:10.1142/S0218213004001922.

[63] G. Sartor, D. Walton, F. Macagno et al., Argumentation schemes for statutory interpretation: A logical analysis, in: Frontiers in Artificial Intelligence and Applications, R. Hoekstra, ed., IOS Press, Amsterdam, Netherlands, 2014, pp. 11-20.

[64] E. Schiappa, The Beginnings of Rhetorical Theory in Classical Greece, Yale University Press, New Haven \& London, 1999.

[65] J. Schneider, K. Samp, A. Passant et al., Arguments about deletion, in: Proceedings of the 2013 Conference on Computer Supported Cooperative Work - CSCW'13, ACM Press, New York, NY, 2013, pp. 1069-1080.

[66] N. Slonim, Y. Bilu, C. Alzate et al., An autonomous debating system, Nature 591 (2021), 379-384. doi:10.1038/s41586021-03215-w.

[67] R. Thomas, J. Masthoff and N. Oren, Is argumessage effective? A critical evaluation of the persuasive message generation system, in: Persuasive Technology: Development of Persuasive and Behavior Change Support Systems, H. OinasKukkonen, K.T. Win, E. Karapanos et al., eds, Springer, Cham, Switzerland, 2019, pp. 87-99. doi:10.1007/978-3-03017287-9_8.

[68] R. Thomas, N. Oren and J. Masthoff, ArguMessage: A system for automation of message generation using argumentation schemes, in: Proceedings of AISB Annual Convention 2018, Curran, New York, NY, pp. 27-31.

[69] R.J. Thomas, M. Collinson and J. Masthoff, Caught by phishing emails? How can argumentation schemes be used to protect users?, in: Proceedings of AISB Annual Convention 2018, Symposium on Digital Behaviour Intervention for Cyber Security, 2018, pp. 1-4.

[70] C. Tindale, Reason's Dark Champions: Constructive Strategies of Sophistical Argument, University of South Carolina Press, Columbia, 2010.

[71] P. Tolchinsky, S. Modgil and U. Cortés, Argument schemes and critical questions for heterogeneous agents to argue over the viability of a human organ for transplantation, in: AAAI 2006 Spring Symposium Series: Argumentation for Consumers of Healthcare, AAAI Press, Stanford, CA, pp. 105-111.

[72] A. Toniolo, T. Norman and K. Sycara, An empirical study of argumentation schemes for deliberative dialogue, in: ECAI 2012, L. De Raedt, C. Bessiere, D. Dubois et al., eds, IOS Press, Amsterdam, Netherlands, 2012, pp. 756-761.

[73] A. Toniolo, T.J. Norman and K. Sycara, Argumentation schemes for collaborative planning, in: Agents in Principle, Agents in Practice. PRIMA 2011, D. Kinny, J.Y. Hsu, G. Governatori et al., eds, Springer, Berlin, Germany, 2011, pp. $323-335$. doi:10.1007/978-3-642-25044-6_26.

[74] S. Toulmin, The Uses of Argument, Cambridge University Press, Cambridge, UK, 1958.

[75] B. Verheij, Artificial argument assistants for defeasible argumentation, Artif Intell 150 (2003), 291-324 (Verheij, $2003 \mathrm{a})$. doi:10.1016/S0004-3702(03)00107-3.

[76] B. Verheij, Dialectical argumentation with argumentation schemes: An approach to legal logic, Artif Intell Law 11 (2003), 167-195 (Verheij, 2003b). doi:10.1023/B:ARTI.0000046008.49443.36.

[77] B. Verheij, Artificial intelligence as law: Presidential address to the seventeenth international conference on artificial intelligence and law, Artif Intell Law 28 (2020), 181-206. doi:10.1007/s10506-020-09266-0.

[78] J. Visser, J. Lawrence, J. Wagemans et al., Revisiting computational models of argument schemes: Classification, annotation, comparison, in: Computational Models of Argument, S. Modgil, K. Budzynska and J. Lawrence, eds, IOS Press, Amsterdam, Netherlands, pp. 313-324.

[79] D. Walton, What is reasoning? What is an argument?, J Philos 87 (1990), 399-419. doi:10.2307/2026735. 
[80] D. Walton, Argumentation Schemes for Presumptive Reasoning, Lawrence Erlbaum Associates, Inc, Mahwah, NJ, 1996.

[81] D. Walton, Argument from appearance: A new argumentation scheme, Log Anal 49 (2006), 319-340.

[82] D. Walton, How to make and defend a proposal in a deliberation dialogue, Artif Intell Law 14 (2006), 177-239. doi:10. 1007/s10506-006-9025-x.

[83] D. Walton, A dialogue model of belief, Argument Comput 1 (2010), 23-46. doi:10.1080/19462160903494576.

[84] D. Walton, Argument mining by applying argumentation schemes, Stud Log 4 (2011), 38-64.

[85] D. Walton, Using argumentation schemes for argument extraction: A bottom-up method, Int J Cogn Informatics Nat Intell 6 (2012), 33-61. doi:10.4018/jcini.2012070103.

[86] D. Walton, Argumentation schemes for argument from analogy, in: Systematic Approaches to Argument by Analogy, H. Ribeiro, ed., Springer, Amsterdam, Netherlands, 2014, pp. 23-40.

[87] D. Walton and T. Gordon, The Carneades model of argument invention, Pragmat Cogn 20 (2012), 1-26. doi:10.1075/pc. 20.1.01wal.

[88] D. Walton and M. Koszowy, From text to scheme: Problems in identifying arguments from expert opinion, Int Rev Pragmat 11 (2019), 109-136. doi:10.1163/18773109-201810015.

[89] D. Walton and F. Macagno, A classification system for argumentation schemes, Argument Comput 6 (2015), 219-245. doi:10.1080/19462166.2015.1123772.

[90] D. Walton, T. Oliveira, K. Satoh et al., Argumentation analytics for treatment deliberations in multimorbidity cases: An introduction to two artificial intelligence approaches, Topoi 40 (2021), 373-386. doi:10.1007/s11245-020-09701-6.

[91] D. Walton, C. Reed and F. Macagno, Argumentation Schemes, Cambridge University Press, New York, NY, 2008.

[92] D. Walton, G. Sartor and F. Macagno, An argumentation framework for contested cases of statutory interpretation, Artif Intell Law 24 (2016), 51-91. doi:10.1007/s10506-016-9179-0.

[93] D. Walton and A. Toniolo, Deliberation, practical reasoning and problem-solving, in: Argumentation, Objectivity, and Bias: Proceedings of the 11th International Conference of the Ontario Society for the Study of Argumentation (OSSA), 18-21 May 2016th, P. Bondy and L. Benacquista, eds, OSSA, Windsor, ON, 2016, pp. 1-19.

[94] D. Walton, A. Toniolo and T. Norman, Missing phases of deliberation dialogue for real applications, in: Proceedings of the 11th International Workshop on Argumentation in Multi-Agent Systems, W. van der Hoek, L. Padgham, V. Conitzer et al., eds, International Foundation for Autonomous Agents and Multiagent Systems, Richland, SC, 2014, pp. 1-20.

[95] V. Wei Feng and G. Hirst, Classifying arguments by scheme, in: Proceedings of the 49th Annual Meeting of the Association for Computational Linguistics: Human Language Technologies - Volume 1, Association for Computational Linguistics, Stroudsburg, PA, 2011, pp. 987-996.

[96] S. Wells, Towards argumentative dialogue as a humane interface between people and intelligent machines, in: Proceedings of the SICSA Workshop on Reasoning, Learning and Explainability, K. Martin, N. Wiratunga and L. Smith, eds, CEUR, New York, NY, 2018.

[97] Y. Zagorulko, O. Domanov, A. Sery et al., Analysis of the persuasiveness of argumentation in popular science texts, in: Artificial Intelligence 18th Russian Conference, RCAI 2020, Moscow, Russia, S. Kuznetsov, A. Panov and K. Yakovlev, eds, Springer, Cham, Switzerland, pp. 351-367.

[98] Y. Zagorulko, N. Garanina, A. Sery et al., Ontology-based approach to organizing the support for the analysis of argumentation in popular science discourse BT - artificial intelligence, in: Artificial Intelligence. RCAI 2019. Communications in Computer and Information Science, S.O. Kuznetsov and A.I. Panov, eds, Springer, Cham, Switzerland, 2019, pp. 348-362. 